\title{
Protective properties of the cultured stem cell proteome studied in an animal model of acetaminophen-induced acute liver failure
}

\author{
Andrey Alexandrovich Temnov ${ }^{1,2} \cdot$ Konstantin Arkadevich Rogov $^{3} \cdot$ Alla Nikolaevna Sklifas $^{1}$. \\ Elena Valerievna Klychnikova ${ }^{4} \cdot$ Markus Hartl $^{5} \cdot$ Kristina Djinovic-Carugo $^{5} \cdot$ Alexej Charnagalov $^{6}$ (D)
}

Received: 19 December 2018 / Accepted: 14 March 2019 / Published online: 12 April 2019

(c) The Author(s) 2019

\begin{abstract}
Chronic overuse of common pharmaceuticals, e.g. acetaminophen (paracetamol), often leads to the development of acute liver failure (ALF). This study aimed to elucidate the effect of cultured mesenchymal stem cells (MSCs) proteome on the onset of liver damage and regeneration dynamics in animals with ALF induced by acetaminophen, to test the liver protective efficacy of MSCs proteome depending on the oxygen tension in cell culture, and to blueprint protein components responsible for the effect. Protein compositions prepared from MSCs cultured in mild hypoxic $\left(5 \%\right.$ and $10 \% \mathrm{O}_{2}$ and normal $\left(21 \% \mathrm{O}_{2}\right)$ conditions were used to treat ALF induced in mice by injection of acetaminophen. To test the effect of reduced oxygen tension in cell culture on resulting MSCs proteome content we applied a combination of high performance liquid chromatography and mass-spectrometry (LC-MS/MS) for the identification of proteins in lysates of MSCs cultured at different $\mathrm{O}_{2}$ levels. The treatment of acetaminophen-administered animals with proteins released from cultured MSCs resulted in the inhibition of inflammatory reactions in damaged liver; the area of hepatocyte necrosis being reduced in the first $24 \mathrm{~h}$. Compositions obtained from MSCs cultured at lower $\mathrm{O}_{2}$ level were shown to be more potent than a composition prepared from normoxic cells. A comparative characterization of protein pattern and identification of individual components done by a cytokine assay and proteomics analysis of protein compositions revealed that even moderate hypoxia produces discrete changes in the expression of various subsets of proteins responsible for intracellular respiration and cell signaling. The application of proteins prepared from MSCs grown in vitro at reduced oxygen tension significantly accelerates healing process in damaged liver tissue. The proteomics data obtained for different preparations offer new information about the potential candidates in the MSCs protein repertoire sensitive to oxygen tension in culture medium, which can be involved in the generalized mechanisms the cells use to respond to acute liver failure.
\end{abstract}

Keywords Acute liver failure $\cdot$ Stem cells $\cdot$ Conditioned medium $\cdot$ Acetaminophen $\cdot$ Proteome $\cdot$ Hypoxia

\begin{tabular}{l}
$\begin{array}{l}\text { Abbreviations } \\
\text { ALF }\end{array}$ \\
$\begin{array}{l}\text { MSCs } \\
\text { NC-MSCs }\end{array}$ \\
$\begin{array}{l}\text { Mesenchymal stem cells } \\
\text { (21\% oxygen) }\end{array}$ \\
HC-MSCs $\quad \begin{array}{l}\text { MSCs cultured in conditions of hypoxia } \\
\text { (10\% or 5\% oxygen) }\end{array}$ \\
$\begin{array}{l}\text { Electronic supplementary material The online version of this } \\
\text { article (https://doi.org/10.1007/s11033-019-04765-z) contains } \\
\text { supplementary material, which is available to authorized users. }\end{array}$ \\
\hline $\begin{array}{l}\text { Alexej Charnagalov } \\
\text { alexej.charnagalov @ist.ac.at }\end{array}$ \\
Extended author information available on the last page of the article
\end{tabular}

LC-MS/MS Liquid Chromatography-Mass Spectrometry and Liquid Chromatography-Tandem Mass Spectrometry

HIF Hypoxia inducible factor

AST Aspartate transaminase

ALT

VEGF

FGF

HGF

IGF

IL6, IL8

DMEM-FCS

Alanine transaminase

Vascular endothelial growth factor

Fibroblast growth factor

Hepatocyte growth factor

Insulin-like growth factor

Interleukins 6 and 8, respectively

Dulbecco's Modified Eagle's Medium supplemented with $10 \%$ fetal calf serum 


\section{Introduction}

Acute liver failure (ALF) caused by the intake of common pharmaceutical drugs is a well-known clinical problem worldwide. There are two major cases when the ALF can emerge in an individual. First, if the person taking the drug in therapeutic doses belongs to the group of so called "oversensitive" patients (on average, one out of 10,000 patients), this person can develop symptoms of liver damage, which vary from mild, transient liver disease to severe organ dysfunction, ending with ALF [1]. As shown in several clinical studies, individual susceptibility to drugs depends not only from the presence and activity of genes participating in the metabolism of xenobiotics but also from functional activity of other genes regulating protective (primarily immune) systems of the organism [2].

Another reason for the development of ALF is intentional or accidental overuse of the drug by "normal", non-"oversensitive" individuals. The most clinically significant drug in this category is acetaminophen (APAP, panadol, tylenol, paracetamol). For example, in the United States 78,000 cases of acetaminophen overdose and about 500 deaths are recorded annually [3]. The hepatotoxicity caused by the overuse of acetaminophen is the most common cause of ALF and accounts for about $50 \%$ of all clinical cases [4].

In animal models, ALF caused by the hepatotoxic effect of acetaminophen was effectively treated with transplanted stem cells [5, 6]. In a number of studies, MSCs have been shown to possess anti-inflammatory, anti-apoptotic and proliferative effects in the liver $[7,8]$. The mechanism of the healing effect of MSCs on the liver is disputed. Several studies indicate that the tissue regeneration is significantly enhanced after stem cell transplantation, the effect being primarily attributed to various paracrine factors released by MSCs. Thus, it was shown that the treatment of the liver with pre-conditioned medium obtained from cultivated MSCs is only marginally inferior to the application of proper MSCs [9]. It should be noted, that MSCs are by nature very sensitive to changes of oxygen concentration because of hypoxic conditions in their habitat, bone marrow. Therefore, as an approach of improving clinical effectiveness in the transplantation of MSCs for the treatment of ALF, careful selection of cultivation conditions in vitro, especially the level of $\mathrm{O}_{2}$ and $\mathrm{CO}_{2}$, is desirable.

\section{Materials and methods}

\section{Animals}

CD-1 male mice (body weight 20-22 g) were kept in the animal facility of the Institute of Cell Biophysics RAS (Pushchino, Russia). Animals were given food and water ad libitum: $24 \mathrm{~h}$ before treatment with acetaminophen and MSCs they received only water. The maintenance and treatment of laboratory animals was done in accordance with the European Convention for the Protection of Vertebrate animals used for experimental and other scientific purposes (No. 123 of 18.03.1986, Council of Europe) and the Guidelines for working with laboratory animals in the Institute of Cell Biophysics (No. 39 of 30.12.2018, ICB Russian Academy of Sciences).

\section{Preparation and characterization of MSCs}

Primary MSCs were extracted from femur bone marrow of CD-1 mice under general anesthesia. The mononuclear fraction of bone marrow cells was isolated by centrifugation in a density gradient of Lympholyte-H (Cedarlane, Canada). The resulting suspension of mononuclear cells was transferred to Petri dishes and cultivated in Dulbecco's Modified Eagle's Medium (DMEM) containing 10\% fetal calf serum (DMEM-FCS).

To confirm that the isolated primary cells possess the properties of MSCs, their ability to undergo osteogenic, chondrogenic and adipogenic differentiation was checked by conventional methods [10]. Osteogenic differentiation was performed in DMEM-FCS with $100 \mu \mathrm{M}$ dexamethasone, $0.1 \mathrm{mM}$ ascorbic acid and $10 \mathrm{nM} \beta$-glycerophosphate and confirmed by the presence of alkaline phosphatase (AP) in cell culture using AP detection kit (SigmaAldrich). Chondrogenic differentiation was done in DMEM-FCS with $100 \mu \mathrm{M}$ of dexamethasone and $0.1 \mu \mathrm{g} /$ $\mathrm{ml}$ TGF- $\beta$ and confirmed by staining with alcian blue. Adipogenic differentiation was checked in DMEM-FCS with $10 \mu \mathrm{M}$ dexamethasone, $10 \mu \mathrm{g} / \mathrm{ml}$ insulin, and $100 \mu \mathrm{g} / \mathrm{ml}$ of 3-isobutyl-1-methylxantine (IBMX) and confirmed by staining with Oil Red-O.

The cultivation of the cell monolayer prepared from suspension of primary MSCs was performed either under the conditions of normoxia $\left(21 \% \mathrm{O}_{2}, 5 \% \mathrm{CO}_{2}\right)$ or hypoxia. For each condition, after the monolayer formation was completed, the medium was exchanged with fresh DMEM-FCS and monolayer was cultivated for 3 days; the culture medium was then harvested and combined with soluble fraction of cell lysate prepared by ultrasonication of respective MSCs monolayer. The resulting conditioned 
media obtained from MSCs cultured in conditions of normoxia (NC-MSCs) or in hypoxic conditions (HC-MSCs) were sterile filtered and used for animal experiments.

\section{Model of acute liver failure}

CD-1 male mice weighing 20-22 g were divided into two groups. Animals in both groups were intraperitoneally administered with $350 \mathrm{mg} / \mathrm{kg}$ acetaminophen. Then animals in control group (group No. $1, \mathrm{n}=15$ ) received $0.5 \mathrm{ml}$ of pure DMEM-FCS, whereas animals of experimental group (group No.2, $\mathrm{n}=15$ ) were administered with $0.5 \mathrm{ml}$ of NC-MSC or HC-MSC compositions (protein concentration $10 \mathrm{mg} / \mathrm{ml}$ ).

\section{Histology of liver specimens}

Materials for histological examination were taken from animals after 4, 24 and $48 \mathrm{~h}$ after injection of acetaminophen. For the assessment of morphological changes, paraffin sections ( $0.3 \mu \mathrm{m}$ thick) were prepared from liver fixed in buffered $10 \%$ formalin.

Assessment of dystrophic and necrotic changes in the liver was determined by a blind semi-quantitative method and expressed in points [11], using a matrix of 10 fields of view. Scores were assigned as follows: 0 - no change; 0.5 - degenerative changes of less than $1 / 3$ of the hepatocytes; 1 - more than $1 / 3$ hepatocytes damaged; 2 - more than 2/3 hepatocytes damaged; $3-100 \%$ hepatocytes damaged; 4 - drug combination dystrophy with sporadic focal necrosis; 5 - extensive focal necrosis. When scoring, biopsy specimens taken from six animals in each of two groups were studied.

\section{Biochemical assays}

The level of liver enzymes aspartate transaminase (AST) and alanine transaminase (ALT) in serum was determined by kinetic method on the biochemical analyzer "Olympus AU2700" (Beckman Coulter, USA). The biochemical effect of the NC-MSC and HC-MSC compositions was evaluated as a function of the level of ALT and AST in the serum of treated animals after different time intervals $(4,24,48 \mathrm{~h})$. The level of ALT and AST determined in intact animals prior to the administration of acetaminophen was used as a control. The level of cytokines and growth factors were determined using ELISA kits from R\&D Systems.

\section{Mass spectrometry analysis of MSC proteins}

For the LC-MS/MS analysis of the whole-cell proteome, harvested cells were washed with fresh DMEM to remove the excess of FCS. Protein samples were precipitated with four volumes cold acetone overnight at $-20{ }^{\circ} \mathrm{C}$. Pellets were re-dissolved in $8 \mathrm{M}$ urea with $50 \mathrm{mM}$ ammonium bicarbonate, the samples were then reduced by incubation with $10 \mathrm{mM}$ dithiothreitol (30 $\mathrm{min}$ at RT) and alkylated with $20 \mathrm{mM}$ iodoacetamide (30 min at RT). The remaining iodoacetamide was quenched with $5 \mathrm{mM}$ dithiothreitol, the samples were then diluted to reduce the urea concentration to 1 M. $2 \mu \mathrm{g}$ trypsin (Trypsin Gold, Promega) was added to each sample. The samples were incubated overnight at $37^{\circ} \mathrm{C}$, the reaction was stopped with $1 \%$ trifluoroacetic acid and the peptides were desalted using $\mathrm{C} 18$ SepPak columns (Waters) according to the manufacturer's instructions.

Peptides were separated on the Ultimate 3000 RSLC nano-flow chromatography system (Thermo-Fisher), using a pre-column for sample loading (Acclaim PepMap C18, $2 \mathrm{~cm} \times 0.1 \mathrm{~mm}, 5 \mu \mathrm{m}$, Thermo-Fisher), and a C18 analytical column (Acclaim PepMap C18, $50 \mathrm{~cm} \times 0.75 \mathrm{~mm}, 2 \mu \mathrm{m}$, Thermo-Fisher), a segmented linear gradient from 2 to $80 \%$ solvent B $(80 \%$ acetonitrile, $0.1 \%$ formic acid; solvent A $0.1 \%$ formic acid) at a flow rate of $230 \mathrm{~nL} / \mathrm{min}$ over $240 \mathrm{~min}$ was applied. Eluted peptides were analyzed on a Q Exactive Plus Orbitrap mass spectrometer (Thermo Fisher), which was connected to the column with a Proxeon nano-spraysource (Thermo Fisher) using coated emitter tips (New Objective).

\section{Proteomics data analysis}

Raw data were processed using the MaxQuant software package (version 1.5.3.12) [12] and the Uniprot mouse reference proteome (http://www.uniprot.org) as well as the database of most common contaminants included in MaxQuant. The search was performed with full trypsin specificity and a maximum of two missed cleavages at a protein and peptide spectrum match false discovery rate of $1 \%$. Carbamidomethylation of cysteine residues were set as fixed, oxidation of methionine and $\mathrm{N}$-terminal acetylation as variable modifications. For label-free quantification (LFQ) the LFQ function [13] and "match between runs" were activated-all other parameters were left at default.

MaxQuant search results were further processed using the Perseus software package [14]. Contaminants, reverse hits, proteins identified only by site, and proteins with less than two razor peptides or less than two MS/MS counts were removed. The $\log 2$ transformed LFQ values were used for relative protein quantification. Proteins with missing LFQ values, representing low abundant and low coverage hits, were removed from further analysis. GO terms and the $\log 2$ LFQ difference (hypoxia - control) was used to calculate a 1D annotation enrichment in Perseus [15].

Statistical data analysis was performed using Statistica 6.0. For nonparametric data, the U-Mann-Whitney test was used. The differences were considered statistically significant when $\mathrm{p}<0.05$. 


\section{Results}

\section{Examination of liver damage caused by acetaminophen}

Administration of acetaminophen leads to profound changes in the central parts of lobules. After $4 \mathrm{~h}$, one-half to two-thirds of the lobule is spanned by the field of damaged hepatocytes in the form of severe protein and fatty degeneration and necrosis of individual cells or groups of them (Fig. 1a). Furthermore, the necrotic cells, surrounding central veins, reveal significant destruction of cellular membranes, phenomena of karyopyknosis and karyorrhexis as well as changes of the trabecular structure in the liver tissue up to fully discomplexed hepatocytes. The central veins and adjacent sinusoids become sharply dilated and filled with erythrocytes or plasma.

One day after the acetaminophen administration (Fig. 1b, c), a pronounced fatty and protein dystrophy of hepatocytes, resulting from the damage of their nuclei in the form of margination of condensed chromatin, is identified across the whole area of the lobules. The least damaged cells are present in areas closed to the portal tracts. The tissues surrounding central veins display hydropic degeneration of hepatocytes with focal vacuolization and lysis of the cytoplasm, disintegration of cell membranes and destruction of nuclei (karyorrhexis, karyopyknosis, karyolysis). The areas containing necrotic hepatocytes are marked by the inflammatory infiltration with a predominance of polymorphonuclear leukocytes and the presence of macrophages (possibly due to the proliferation of Kupffer cells). The accumulation of polymorphonuclear leukocytes was also noted in the lumen of some central veins along with pronounced discomplexation of hepatocytes. However, in the vicinity of the portal tracts, some preserved areas of the trabeculae are revealed.

Two days after the administration of acetaminophen (Fig. 1d), a widespread discomplexation of hepatocytes is revealed in the liver tissues. Trabecula sites are identified only near some of the portal tracts. In hepatocytes, a pronounced large - and medium-drop fatty degeneration of cells predominates and margination of chromatin, most developed in the central parts of the lobules, occurs. Only
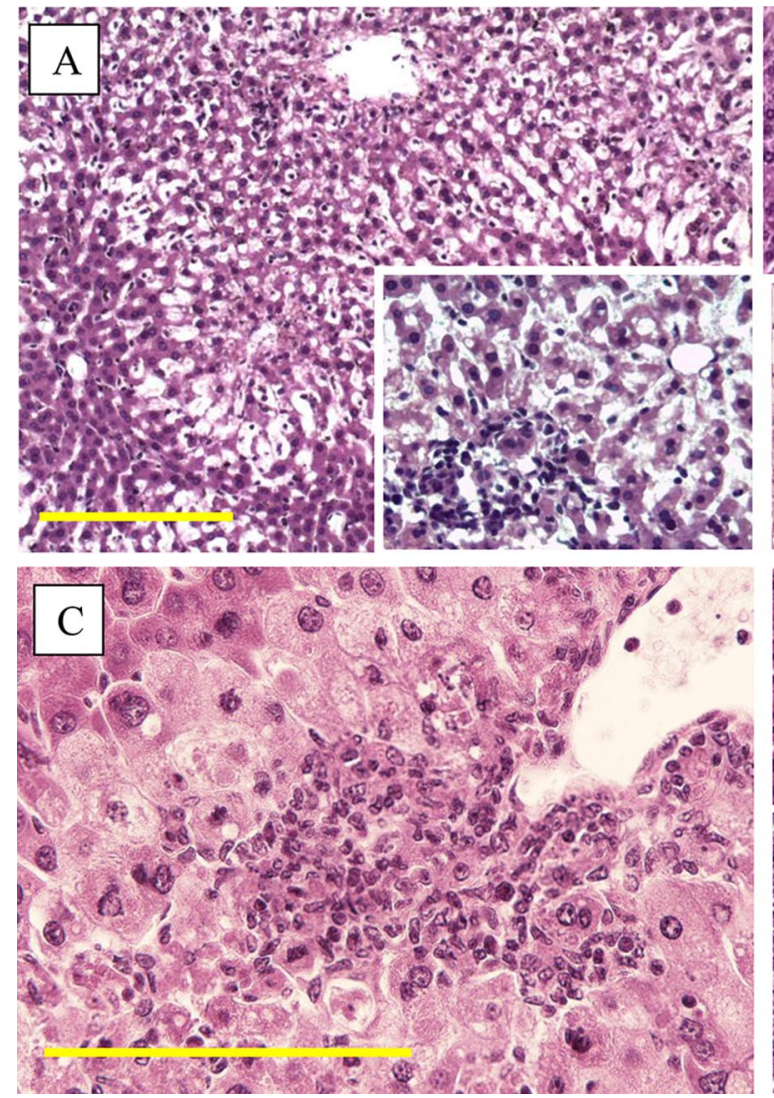

Fig. 1 Histology of mouse liver after acetaminophen injection. Animals were injected with $350 \mathrm{mg} / \mathrm{kg}$ acetaminophen, specimens were stained with hematoxilin- eosin. a $4 \mathrm{~h}$ after the injection; b $24 \mathrm{~h}$ after

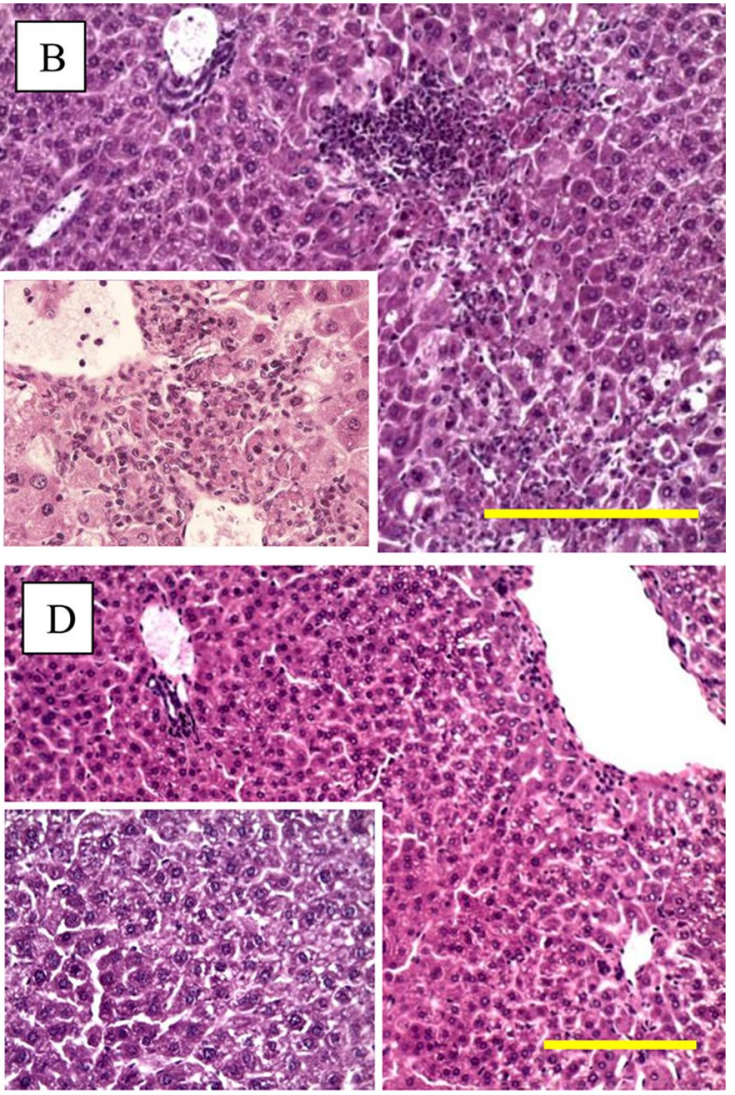

injection; c $24 \mathrm{~h}$ after injection ; d $48 \mathrm{~h}$ after injection. Yellow bars correspond to $100 \mu \mathrm{m}$. Magnification on inserts is twice as high compared to main pictures. (Color figure online) 

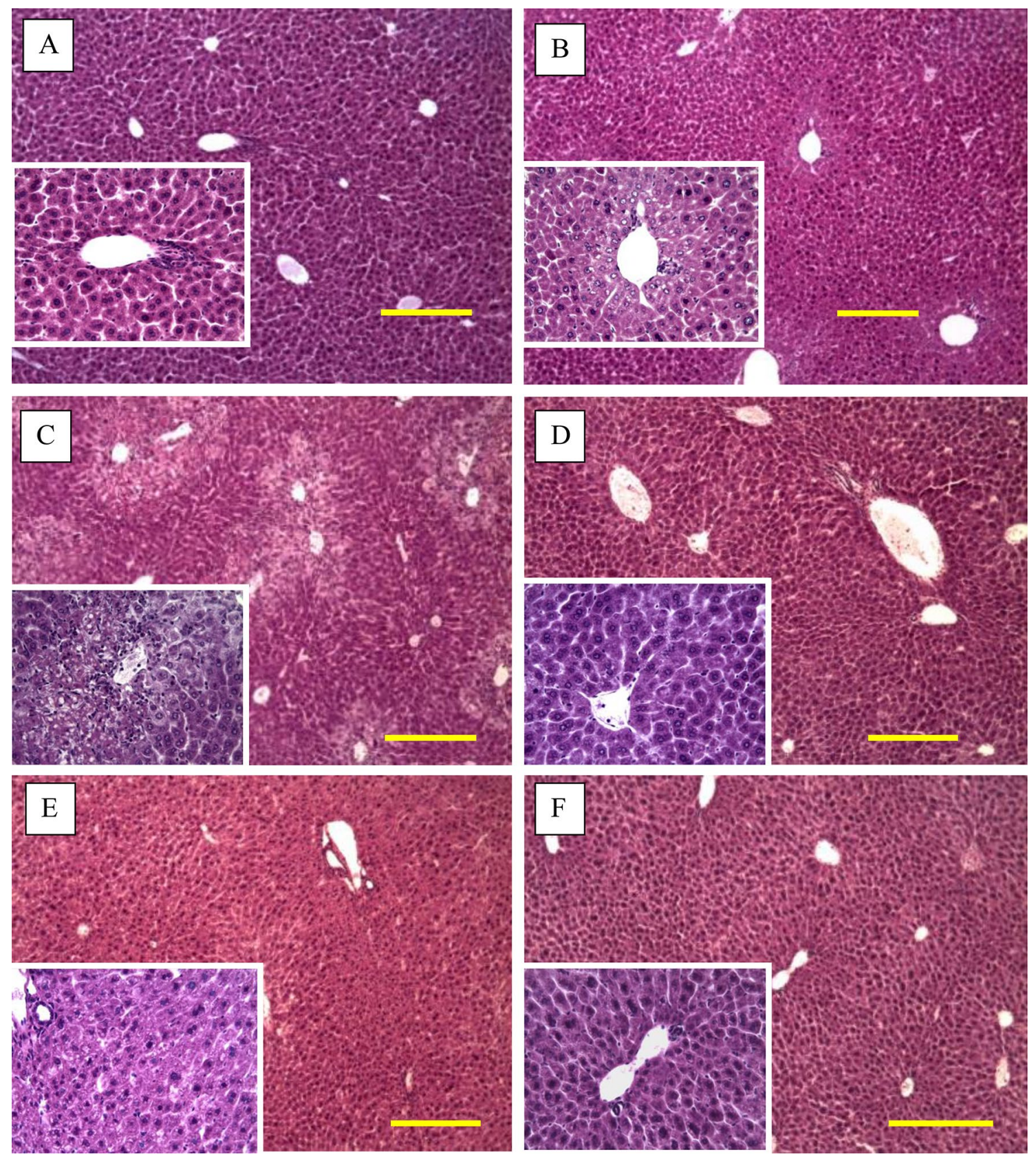

Fig. 2 Histology of mouse liver after acetaminophen injection and simultaneous treatment with MSCs protein compositions. Animals were injected with $350 \mathrm{mg} / \mathrm{kg}$ acetaminophen, specimens were stained with hematoxilin- eosin. Magnification on inserts is twice as high compared to main pictures. a $4 \mathrm{~h}$ after the injection and treatment with NC- MSCs; b $4 \mathrm{~h}$ after the injection and treatment with

HC-MSCs; $\mathbf{c} 24 \mathrm{~h}$ after the injection and treatment with NC-MSCs; d $24 \mathrm{~h}$ after the injection and treatment with HC-MSCs; e $48 \mathrm{~h}$ after the injection and treatment with NC-MSCs; $f 48 \mathrm{~h}$ after the injection and treatment with HC-MSCs. Yellow bars correspond to $100 \mu \mathrm{m}$. (Color figure online)

hepatocytes closed to the portal tract remain minimally damaged. Inflammatory infiltration is insignificant at this

point and is mainly determined near portal tracts. In the lobules, expanded sinusoids are noted. 


\section{Protective effect of MSC proteins}

The administration of MSCs compositions into animals largely improved the histological picture of the hepatic tissue. Within $4 \mathrm{~h}$ after acetaminophen administration, the detected liver tissue lesions in MSC-treated animals are significantly less than in the control group.

Thus, $4 \mathrm{~h}$ after the simultaneous administration of acetaminophen and NC-MSCs (Fig. 2a), the liver segments still retain a trabecular structure. There are no signs of venous plethora and inflammatory infiltration. On the other hand, the swelling of endothelial cells of sinusoids was noted. The hepatocyte lesions do not have a distinct zonality and consist in dystrophic changes in the cytoplasm in the form of protein and small droplet fatty degeneration. In a few cells, the destruction of the cytolemma was noted. However, throughout the area of the lobules, a few hepatocytes with large hyperchromic nuclei containing fine dispersed chromatin and binuclear cells were identified.

The histology status was further improved when the HCMSC composition was injected to animals (Fig. 2b). After $4 \mathrm{~h}$, acetaminophen-induced damage to the hepatic tissue remains minimal. There was a violation of the trabecular structure of the lobules with focal discomplexation of hepatocytes. Pulmonary and small droplet fatty degeneration of hepatocytes, mainly around the central vein or in the 3rd zone of the acini, where cell obesity acquires a large droplet character was detected, but there were no signs of circulatory disturbance and inflammatory infiltration. In the sinusoids, the number of Kupffer cells is increased, which may be an indirect sign of their proliferation. In hepatocytes (without pronounced zoning), large hyperchromic nuclei containing fine dispersed uniformly distributed chromatin and damaged nuclei alternate with coarse dispersed chromatin with a tendency to margination. Margination of chromatin is most noticeable in cells surrounding the central veins and in the $3 r d$ zone of the acinus.

Twenty-four hours after the joint administration of acetaminophen and NC-MSC (Fig. 2c), an increase in the degree of hepatocyte damage is observed in comparison with the 4-h exposure period, however, the severity and prevalence of dystrophic changes are significantly less than in the control group. The trabecular structure is preserved in the periportal zones of the lobules. There was no stagnant plethora. In the centrolobular areas, pronounced tissue lesions in forms of protein, large-droplet fat and hydrophilic degeneration, up to partial and monocellular necrosis of hepatocytes or their groups, were observed. In areas of necrotic changes, insignificant infiltration of polynuclear leukocytes and macrophages can be seen.

When the HC-MSC composition was administered to acetaminophen treated animals, the degree of liver damage was also increased at $24 \mathrm{~h}$ relative to $4 \mathrm{~h}$ (Fig. 2d). The damage varies from animal to animal but one can distinguish the following common features: the trabecular structure of the lobules is preserved (discomplexation occurs only in the centrolobular zones of individual lobules). A large-droplet fatty degeneration of hepatocytes and monocellular necrosis is also seen. The nuclei of the cells of this zone are marked by the margination of coarse dispersed chromatin. In other parts of the lobules, there are binuclear cells, hepatocytes with large hyperchromic nuclei, rich in uniformly distributed fine dispersed chromatin, and single mitoses, but no signs of inflammatory infiltration and blood circulation disturbances were observed.

Forty-eight hours after the injection of the NC-MSC composition and acetaminophen (Fig. 2e), a distinct recovery of damagedF tissues is observed. The trabecular structure of lobules was preserved and no necrotic hepatocytes were observed. Dystrophic changes in the form of protein, medium- and large-droplet fatty degeneration were, in this case, most pronounced in the centrolobular regions and in the $3 \mathrm{rd}$ zone of the acinus. The margination of coarse dispersed chromatin predominates in these cells, but there is no inflammatory infiltrates and disturbance of the blood circulation. At the same time, the number of cells with large hyperchromic nuclei containing uniformly distributed fine dispersed chromatin and binuclear cells was increasing.

A remarkably different effect was observed for the HCMSC composition injected along with acetaminophen (Fig. 2f). Forty-eight hours after the injection, the trabecular structure of the liver tissue was preserved and there were no morphological changes in blood vessels. Small-droplet fatty degeneration of hepatocytes was noted mainly in the centrolobular and periportal zones and no damage to the

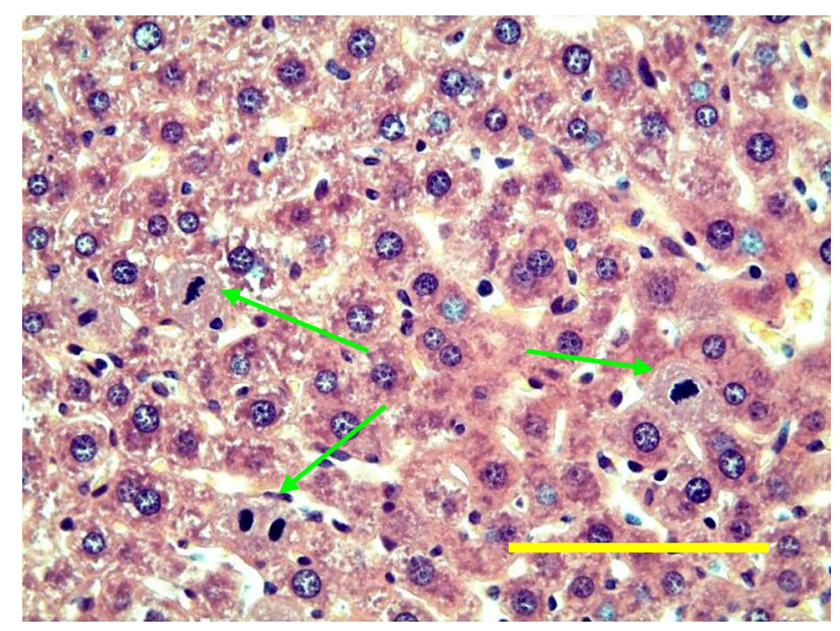

Fig. 3 Histology of mouse liver after the HC-MSC treatment. Specimens were prepared from mouse liver $48 \mathrm{~h}$ after acetaminophen injection $(350 \mathrm{mg} / \mathrm{kg})$ and simultaneous treatment with HC-MSC. Yellow bar corresponds to $100 \mu \mathrm{m}$. Hepatocyte mitoses are shown by green arrows. (Color figure online) 
Table 1 Scoring of dystrophic and necrotic liver changes upon the administration of MSCs compositions

\begin{tabular}{llll}
\hline & $4 \mathrm{~h}$ & $24 \mathrm{~h}$ & $48 \mathrm{~h}$ \\
\hline Control & $2.7 \pm 1.4$ & $2.2 \pm 0.6$ & $1.9 \pm 0.5 \times$ \\
HC-MSC & $0.1 \pm 0.05^{*}$ & $1.0 \pm 0.7^{*}$ & $2.2 \pm 1.1$ \\
NC-MSC & $0.2 \pm 0.05^{*}$ & $1.3 \pm 0.8^{*}$ & $1.0 \pm 0.5^{* \neq}$ \\
\hline
\end{tabular}

The data are summarized from five independent experiments (three animals in each) with five individual sections taken from each animal (about 80-100 different microscopic views of each section was inspected and scored)

*compared to control $(\mathrm{p}<0.05)$

fcompared to HC-MSC at $48 \mathrm{~h}(\mathrm{p}<0.05)$

$\times$ compared to control at $4 \mathrm{~h}(\mathrm{p}<0.05)$

nuclei was detected. On the contrary, the number of cells with large hyperchromic nuclei and binucleated hepatocytes was increased (Fig. 3), suggesting increased protein-synthetic activity.

The effects described above are summarized in the Table 1. Thus, the administration of acetaminophen causes pronounced damage to the hepatic tissue already within the first hours after injection. After $24 \mathrm{~h}$, the level of damage is reduced and by $48 \mathrm{~h}$ there is a reliable recovery in control animals in comparison with the initial period. When an animal received an MSC composition along with acetaminophen, a reliable protective effect is observed within the first $24 \mathrm{~h}$. It should be noted that the compositions obtained from cells cultivated under the conditions of reduced oxygen content (HC-MSCs) have a more pronounced effect during the first $24 \mathrm{~h}$, but when used in $48 \mathrm{~h}$, a significant increase in the number of dystrophically altered hepatocytes is observed.

\section{Effect of MSCs compositions on the biochemical status of liver}

The data from the biochemical tests of common serum liver enzymes, ALT and AST, showed that the MSCs compositions, when administered in the early acute period of liver damage, significantly reduce the level of liver enzymes, the protective effect being especially noticeable at earlier stages of liver damage in animals treated with HC-MSCs (Fig. 4). Thus, $4 \mathrm{~h}$ after administration of acetaminophen, the mean concentration of ALT in the control group (animals treated with acetaminophen but not received any of C-MSC composition) was $2492,0 \pm 203,0 \mathrm{IU} / 1$, in the NC-MSCtreated animals it consisted of 223,2 $\pm 8,4 \mathrm{IU} / 1$, and in the HC-MSC-treated animals the ALT concentration dropped to $48,3 \pm 15,8 \mathrm{IU} / 1$, the last value is close to the ALT level in intact animals.

The mean concentration of AST, $4 \mathrm{~h}$ after acetaminophen administration, was $1213,5 \pm 101,3 \mathrm{IU} / \mathrm{l}$ in the control group, $98,5 \pm 15,1 \mathrm{IU} / 1$ in HC-MSC group, and 621,4 $\pm 23,1 \mathrm{IU} / 1$ in the NC-MSC group. It should be noted that the values of AST and ALT in the HC-MSC group was significantly lower than the level of enzymes in the NC-MSC group. After $24 \mathrm{~h}$, the enzyme levels in the control group decreased, but still

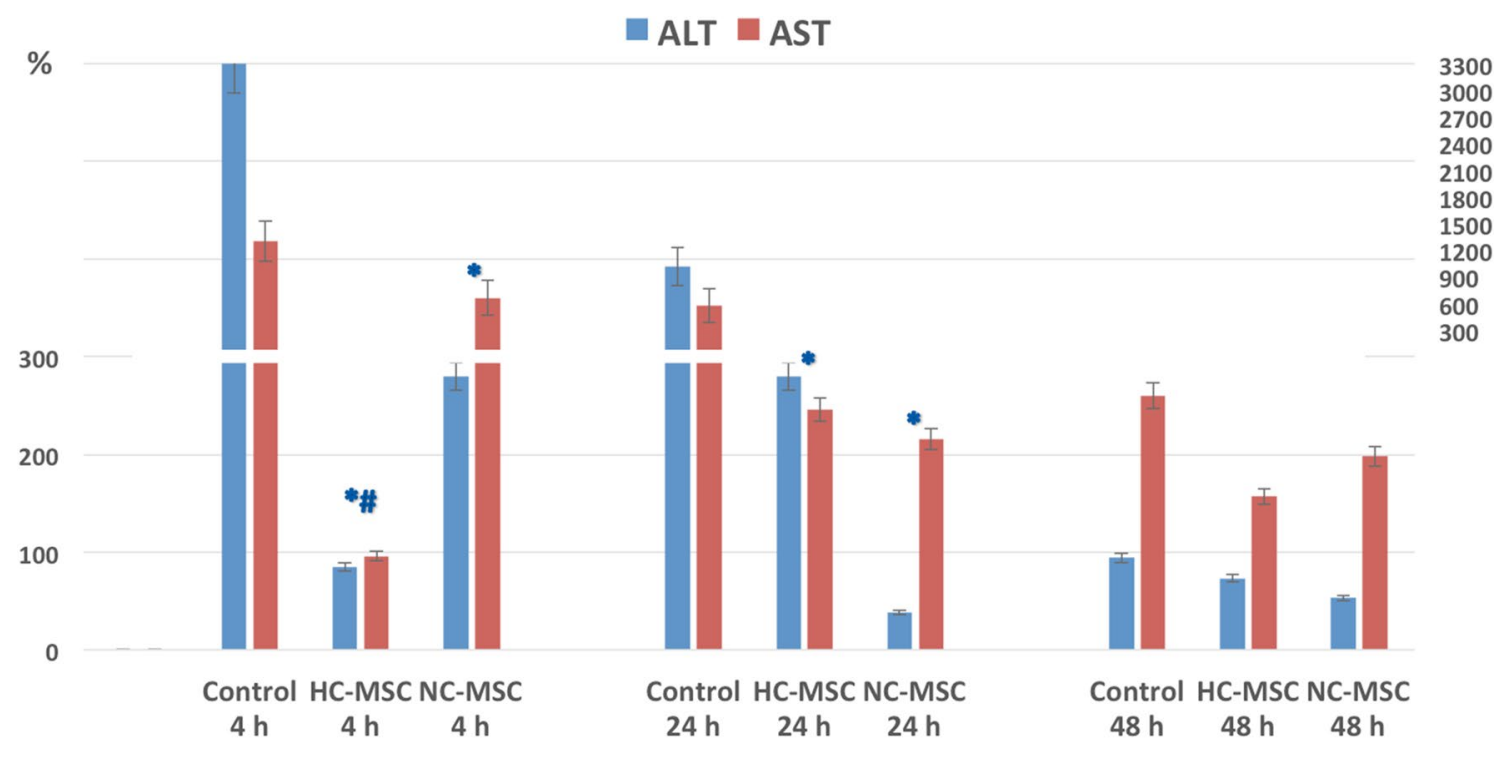

Fig. 4 Biochemical status of liver in animals with acetaminopheninduced ALF. Graph represents relative activities of liver enzymes AST and ALT in control animals with acetaminophen-induced ALF and in the HC- MSC or NC-MSC treated animals at different times after the administration of acetaminophen. The mean concentrations of AST and ALT measured in intact (non-ALF) animals were considered as $100 \%$. The data from three independent experiments with five individual repeats in each are summarized. ${ }^{*} \mathrm{p}<0.05$ to control; $\# \mathrm{p}<0.05$ to NC-MSC 
considerably exceeded the mean ALT and AST concentrations in non-ALF animals. At the same time, the levels of ALT and AST in the HC-MSC group increased as compared to those levels in control animals and NC-MSC group. One of the possible explanations is that the hypoxic environment forces MSC to produce active substances that inhibit the inflammatory process for a while. When the anti-inflammatory agents are inactivated in the liver tissue, the inflammation continues to develop.

This is also evident that after $24 \mathrm{~h}$, the liver enzyme levels remained significantly elevated in the control group, and, contrary, the level of enzymes in the group, which was treated with C-MSCs was approaching normal values. After $48 \mathrm{~h}$ the values for AST and ALT concentrations in two groups are aligned, however, the level of AST remained elevated in all groups.

\section{Change of cytokine level in MSCs at mild hypoxia}

During cultivation in DMEM-FCS, MSCs enrich culture medium with various cytokines and growth factors. Here, to test the differences between biological compositions prepared from cells cultivated at normal $\mathrm{O}_{2}$ concentration (21\%) and cells grown in moderate hypoxic conditions $\left(10 \% \mathrm{O}_{2}\right)$, we determined the concentration of several cell signaling factors in both NC- and HC-MSC variants. As follows from data presented in Table 2, the low level of $\mathrm{O}_{2}$ leads to an increase in the production of the signal molecules relevant to hypoxia.

\section{Proteome differences in MSCs cultured at different $\mathrm{O}_{2}$ levels}

In order to elucidate the difference in the proteome of normal and hypoxic cells and possibly to identify individual proteins which differ in both MSC sources, we further analyzed the protein distribution pattern in whole cell lysates prepared from normoxic and hypoxic MSCs. The SDS-PAGE of MSCs compositions revealed only subtle differences in the intensity of protein bands in the area of gel corresponding to $15-20 \mathrm{kDa}$, the protein bands here being still well resolved (Fig. 5). The area above $30 \mathrm{kDa}$ in samples

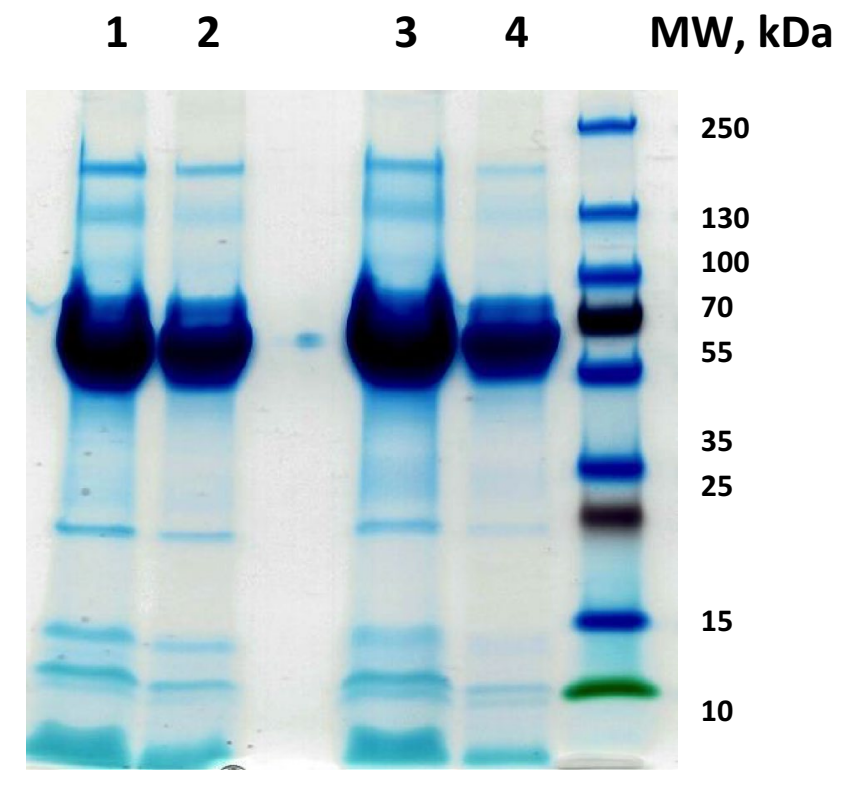

Fig. 5 SDS-PAAG electrophoresis of MSC whole cell extracts. 1 and 3 - NC-MSC composition (cells cultured in monolayer at $21 \% \mathrm{O} 2$ ), 2 - HC-MSC composition (cells cultured at 10\% O2), 4 - HC-MSC composition (cells cultured at 5\% O2). The samples were normalized according to a total protein content using BSA as a standard

of cells grown in DMEM-FCS has a fuzzy background and poorly resolved protein band pattern on a gel because of the presence of abundant DMEM-FCS proteins, e.g. BSA and immunoglobulins, making impossible the accurate identification and quantification of protein bands derived from cells. Nevertheless, the observation in SDS-PAGE gels of small differences between the samples in Coomassie-stained bands at $15-20 \mathrm{kDa}$ suggested potential proteome changes in MSCs upon hypoxia, which were confirmed by LC-MS/MS analysis of whole cell lysates, as described below.

To investigate the effects of hypoxia on the protein distribution in MSCs, we used a shotgun proteomics approach applied to whole cell lysates. We digested whole extracts of HC-MSCs prepared from cells grown at $10 \%$ and $5 \% \mathrm{O}_{2}$ and compared their protein expression pattern with control NC-MSC digests. The same $10 \% \mathrm{O}_{2}$ samples were used to treat experimental animals, so the liver-regenerating potency

Table 2 ELISA determination of cytokines and growth factors in biological compositions prepared from MSCs cultivated in normoxic and hypoxic conditions

\begin{tabular}{lclcc}
\hline & VEGF $(\mathrm{pg} / \mathrm{ml})$ & FGF $(\mathrm{pg} / \mathrm{ml})$ & IL8 $(\mathrm{pg} / \mathrm{ml})$ & IL6 $(\mathrm{pg} / \mathrm{ml})$ \\
\hline DMEM-FCS (control) & $2.3 \pm 0.4$ & 0.0 & $2.7 \pm 1.3$ & $2.7 \pm 0.9$ \\
NC-MSC composition & $1200.0 \pm 388.0^{*}$ & $0.6 \pm 0.4$ & $240.0 \pm 42.5^{*}$ & $250.9 \pm 55.8^{*}$ \\
HC-MSC composition & $1780.3 \pm 234.0^{*}$ & $0.6 \pm 0.3$ & $320.3 \pm 65.4^{*}$ & $400.3 \pm 45.3^{*}$ \\
\hline
\end{tabular}

The data from three independent experiments with five individual repeats in each are summarized.

$* \mathrm{p}<0.01$ to control 
of the samples was confirmed and consistent with histological and biochemical results presented above. In our pilot study we were able to identify more than 2600 proteins and we acquired relative quantitative information for more than 1900 proteins using label-free quantification (summarized in supplemental proteomics data table and figures S1A and S1B). This experiment represents data from single sample replicates with confirmed biological activity and, thus, we did not compare the data on the level of individual proteins but rather assumed that general trends in differential expression might be observable when analyzing the data on a functional level. In a $1 \mathrm{D}$-annotation enrichment analysis on the $\log 2$ protein ratio using gene ontology terms a number of differentially enriched functional categories emerged which suggest adaptive gene regulation in response to different oxygen concentration in the cell cultures (Table 3).

This spot-check probing the MSC proteome suggests complex differences between proteomic fingerprints of normal and hypoxic cells. Although the identified proteins belong to different functional classes or clusters, their overall pattern suggests that, besides changes in the expression pattern of proteins responsible for intracellular transport, energy metabolism and respiration; the differences in the amount of protein-modifying enzymes, transcriptional factors and various intra- and inter-cellular signal molecules, caused by cultivation at low oxygen, might contribute to the healing effect of MSCs compositions in liver.

\section{Discussion}

The positive effect observed in MSC transplantation on models of acute renal and hepatic insufficiency, ischemia-reperfusion injury of various organs, burn lesions, and pulmonary edema has been repeatedly confirmed [16-18]. At the same time, positive clinical effect in MSCs transplantation is typically observed during first hours after administration, thus, indicating that the effect apparently does not depend on the structural changes in tissues caused by transplanted cells, but depends primarily on the paracrine factors including cytokines and growth factors produced by cells and/or short sections of RNA that also have a pronounced regenerative effect $[17,19]$.

However, the level of production of cytokines and growth factors is strongly influenced by conditions of the MSCs' microenvironment. With the decrease in the level of $\mathrm{O}_{2}$, cells begin to actively express the hypoxia inducible factor (HIF), which is one of the key transcription factors involved in the adaptation of MSC to decreased oxygen content. This factor is inactivated with increased oxygen concentration and its content remains stable under hypoxic conditions [19].

Activation of HIF products triggers the transcription of angiogenic genes and stimulates MSC to produce other biologically active molecules such as VEGF, FGF, HGF, IGF, etc. [20-23]. Cultivation conditions significantly influence the production of cytokines and growth factors released by stem cells. Other studies showed a positive effect on the differentiation, lifespan and anti-inflammatory characteristics of MSCs cultured at physiological oxygen level (from 1 to $7 \%$ oxygen) $[24,25]$. In this work the effect of oxygen concentration in the culture medium was studied and the results confirmed the hypothesis that the interplay of paracrinic factors released by MSCs and extracellular signaling during tissue regeneration with transplanted stem cells is important.

The results of the qualitative and quantitative analyses of MSCs compositions suggest differences in protein expression pattern between NC-MSCs and HC-MSCs which may contribute, as single components or in combination, to the therapeutic and healing effects of MSCs.
Table 3 Categories significantly enriched in a $1 \mathrm{D}$ annotation enrichment analysis based on LFQ intensity values and gene ontology (GO) slim terms

\begin{tabular}{lccccc}
\hline GO slim term & Group size & Score & P-value & Benj. Hoch. FDR & $\begin{array}{l}\text { log2 ratio } \\
\text { group mean }\end{array}$ \\
\hline Respiratory chain complex III & 5 & & & & 0.656 \\
Receptor complex & 14 & 0.766 & $7.56 \mathrm{E}-07$ & $1.77 \mathrm{E}-05$ & 0.604 \\
Proton-transporting ATPase complex & 14 & 0.738 & $1.92 \mathrm{E}-06$ & $3.75 \mathrm{E}-05$ & 0.536 \\
NADH dehydrogenase complex & 11 & 0.755 & $1.55 \mathrm{E}-05$ & $2.59 \mathrm{E}-04$ & 0.530 \\
Hydrogen transport & 24 & 0.741 & $4.12 \mathrm{E}-10$ & $3.74 \mathrm{E}-08$ & 0.499 \\
Transmembrane transport & 36 & 0.669 & $5.77 \mathrm{E}-12$ & $1.58 \mathrm{E}-09$ & 0.442 \\
Lipid particle & 16 & 0.536 & $2.20 \mathrm{E}-04$ & $2.34 \mathrm{E}-03$ & 0.321 \\
Nucleoside metabolic process & 19 & -0.501 & $1.66 \mathrm{E}-04$ & $2.06 \mathrm{E}-03$ & -0.307 \\
Ubiquitin ligase complex & 23 & -0.438 & $3.00 \mathrm{E}-04$ & $2.51 \mathrm{E}-03$ & -0.313 \\
Protein polymerization & 12 & -0.616 & $2.28 \mathrm{E}-04$ & $2.40 \mathrm{E}-03$ & -0.353 \\
Transcriptional repressor complex & 9 & -0.617 & $1.39 \mathrm{E}-03$ & $8.57 \mathrm{E}-03$ & -0.374 \\
\hline
\end{tabular}

The following thresholds were applied: $\log 2$ mean LFQ ratio $>0.3$ or $<-0.3$, FDR $<0.01$. For a full list of gene ontology terms in this analysis see supplemental data 
The majority of proteins enriched in HC-MSCs are involved in intracellular transport and cellular respiration. These differences could be well explained by the adaptation of cells to low oxygen concentration and changes of intracellular $\mathrm{pH}$ in response to oxidative stress. On the other hand, the enrichment of proteins belonging to other protein groups, in particular to receptor complexes, seems to be rather specific and relevant to such effects.

Remarkably, by analysis of the whole hypoxic MSC proteome we observed significant enrichment in the groups of proteins which belong to the respiratory supercomplex localized in mitochondria [26], e.g. NADH dehydrogenase complex (complex I), respiratory complex III (also known as cytochrome bc1 complex or cytochrome c oxidoreductase) and ATPases (complex V). Higher supplement of these redox proteins can potentially contribute to protective effect of MSCs, particularly enhancing the utilization of ROS in damaged tissues and protecting membrane integrity. It was shown that disintegration of respiratory chain or its functional deficiency play role in both, p53 and MAPK apoptosis signaling pathways [27, 28].

Deficiencies in the regulation of respiratory chain promote the permeabilisation of cell membranes, which, in turn, leads to the accumulation of oxidized form of cytochrome $c$ in cytoplasm and activation of pro-apoptotic events [29, 30]. Cytochrome $c$ oxidoreductase, which was found in higher proportion in hypoxic MSCs, can compensate rapid release of oxidized cytochrome $c$ in damaged liver cells by keeping cytochrome $\mathrm{c}$ in predominantly reduced state. The impact of redox status of cultured stem cells can nonetheless be not necessarily restricted to the tissue regeneration effects described in this paper. The proteomics results should be regarded as preliminary observations and future quantitative studies will certainly help to reveal the MSCs responses to hypoxia in more details.

One can suggest that MSCs that have been exposed to hypoxia produce significantly more biologically active molecules, including, but not limited to cytokines, growth factors and receptor proteins and, thus, they can be useful to prevent and reverse necrotic changes in liver caused by pharmaceuticals including acetaminophen. Although the clinical relevance of the role of MSC proteins in liver regeneration remains to be studied, this work provides a conclusive proof for the idea to use protein subsets extracted from cultured MSCs instead of transplanted primary MSCs, to cure liver damage caused by ALF. Such approach would potentially facilitate the treatment and reduce the risk of adverse physiological reactions caused by an injection of whole cells.

Furthermore, systematic proteomic and metabolomics studies will help to understand the mechanisms of the protective and healing effect of MSCs compositions on damaged liver tissue and identify corresponding lead molecules.

\section{Conclusions}

The biochemical and histological experiments conducted in this study showed that the MSC protein compositions significantly inhibit the level of inflammatory reaction and reduce the area of hepatocyte necrosis in the first $24 \mathrm{~h}$. Results of mass-spectrometry analysis done on the MSC protein compositions confirm that the amount of oxygen available to growing cells considerably affect their proteome.

Acknowledgements Open access funding provided by Institute of Science and Technology (IST Austria). The work was done with the support from the Austrian Federal Ministry of Economy, Family and Youth through the initiative "Laura Bassi Centres of Expertise" funding the Center of Optimized Structural Studies, grant No. 253275, from the Russian Basic Research Fund, grant No. 17-07-00439, and from the COST action BM1405 - Non-globular proteins - from sequence to structure, function and application in molecular physiopathology (NGP-NET). Authors thank Dr. James A. Letts for discussion and critical proofreading of the manuscript. We would also like to thank the MFPL Mass Spectrometry Facility at the Campus Vienna Biocenter for excellent technical and scientific support for proteomics experiments, and the VBCF for providing the MS instrument pool.

Author contributions AAT performed cultivation of mesenchymal stem cells and preparation of cell compositions. ANS carried out experiments with laboratory animals. KAR participated in the design and validation of histology experiments. EVK carried out biochemical tests. AC performed sample preparation for mass-spectrometry analysis of MSC compositions. MH performed mass-spectrometry analysis of MSC compositions, validated proteomics data and helped to draft the manuscript. KD-C supervised proteomics experiments, and participated in their design and coordination. AAT and AC conceived of the presented idea and drafted the manuscript. All authors read and approved the final manuscript.

Funding The studies were supported by the Austrian Federal Ministry of Economy, Family and Youth through the initiative "Laura Bassi Centres of Expertise" funding the Center of Optimized Structural Studies, grant No. 253275, and by the Russian Basic Research Fund, grant No. 17-07-00439.

\section{Compliance with ethical standards}

Conflict of interest Authors declare that none of them has any conflict of interest or financial arrangements that could potentially influence the described research.

Ethical approval The maintenance and ethical treatment of laboratory animals was done in accordance with Guidelines of the European Convention for the Protection of Vertebrate animals used for experimental and other scientific purposes and the Laws of Russian Federation.

Research involving human and animal participants The studies were performed in mice and did not involve any human participants, human data or human tissues.

Open Access This article is distributed under the terms of the Creative Commons Attribution 4.0 International License (http://creativeco mmons.org/licenses/by/4.0/), which permits unrestricted use, distribution, and reproduction in any medium, provided you give appropriate 
credit to the original author(s) and the source, provide a link to the Creative Commons license, and indicate if changes were made.

\section{References}

1. Fontana RJ (2014) Pathogenesis of idiosyncratic drug-induced liver injury and clinical perspectives. Gastroenterology 146(4):914-928

2. Urban TJ, Shen Y, Stolz A, Chalasani N, Fontana RJ, Rochon $\mathrm{J}$ et al (2012) Limited contribution of common genetic variants to risk for liver injury due to a variety of drugs. Pharmacogenet Genomics 22(11):784-795

3. Budnitz DS, Lovegrove MC, Crosby AE (2011) Emergency department visits for overdoses of acetaminophen-containing products. Am J Prev Med 40(6):585-592

4. Lee WM (2012) Acute liver failure. Semin Respir Crit Care Med 33(1):36-45

5. Salomone F, Barbagallo I, Puzzo L, Li Volti G (2013) Efficacy of adipose tissue-mesenchymal stem cell transplantation in rats with acetaminophen liver injury. Stem Cell Res 11(3):1037-1044

6. Stock P, Brückner S, Winkler S, Dollinger MM, Christ B (2014) Human bone marrow mesenchymal stem cell-derived hepatocytes improve the mouse liver after acute acetaminophen intoxication by preventing progress of injury. Int J Mol Sci 15(4):7004-7028

7. Khubutiya MS, Vagabov AV, Temnov AA, Sklifas AN (2014) Paracrine mechanisms of proliferative, anti-apoptotic and antiinflammatory effects of mesenchymal stromal cells in models of acute organ injury. Cytotherapy 16(5):579-585

8. Montzka K, Fuhrmann T, Muller-Ehmsen J, Woltje M, Brook GA (2010) Growth factor and cytokine expression of human mesenchymal stromal cells is not altered in an in vitro model of tissue damage. Cytotherapy 12(7):870-880

9. Lee JW, Fang X, Gupta N, Serikov V, Matthav MA (2009) Allogeneic human mesenchymal stem cells for treatment of E. coli endotoxin-induced acute lung injury in the ex vivo perfused human lung. Proc Natl Acad Sci USA 106(38):16357-16362

10. Yagi H, Soto-Gutierrez A, Navarro-Alvarez N, Nahmias Y, Goldwasser Y, Kitagawa Y et al (2010) Reactive bone marrow stromal cells attenuate systemic inflammation via sTNFR1. Mol Therapy 18(10):1857-1864

11. Kosyreva AM (2013) Histophysiology of immune system and barrier organs in the late period of orchiectomy in Wistar rats. Bull Exp Biol Med 154(4):480-484

12. Cox J, Mann M (2008) MaxQuant enables high peptide identification rates, individualized p.p.b.-range mass accuracies and proteome-wide protein quantification. Nat Biotechnol 26(12):1367-1372

13. Cox J, Hein MY, Luber C, Paron I, Nagaraj N, Mann M (2014) Accurate proteome-wide label-free quantification by delayed normalization and maximal peptide ratio extraction, termed MaxLFQ. Mol Cell Proteomics 13(9):2513-2526

14. Tyanova S, Temu T, Sinitcyn P, Carlson A, Hein MY, Geiger T et al (2016) The Perseus computational platform for comprehensive analysis of (prote)omics data. Nat Methods 13(9):731-740

15. Cox J, Mann M (2012) 1D and 2D annotation enrichment: a statistical method integrating quantitative proteomics with complementary high-throughput data. BMC Bioinf 13(Suppl 16):S12

16. Kanazawa H, Fujimoto Y, Teratani T, Iwasaki J, Kasahara N, Negishi K et al (2011) Bone marrow-derived mesenchymal stem cells ameliorate hepatic ischemia reperfusion injury in a rat model. PLoS ONE 6(4):e19195

17. Introna $\mathrm{M}$, Imberti $\mathrm{B}$, Corna $\mathrm{D}$, Abbate $\mathrm{M}$, Rota $\mathrm{C}$ et al (2008) Human bone marrow mesenchymal stem cells accelerate recovery of acute renal injury and prolong survival in mice. Stem Cells 26(8):2075-2082

18. Morigi M, Rota C, Montemurro T, Montelatici E, Lo Cicero V, Imberti $\mathrm{V}$ et al (2010) Life-sparing effect of human cord bloodmesenchymal stem cells in experimental acute kidney injury. Stem Cells 28(3):513-522

19. Jaakkola P, Mole DR, Tian YM, Wilson MI, Gielbert J, Gaskell SJ et al (2001) Targeting of HIF-alpha to the von Hippel-Lindau ubiquitylation complex by $\mathrm{O}_{2}$-regulated prolyl hydroxylation. Science 292(5516):468-472

20. Ahluwalia A, Tarnawski AS (2012) Critical role of hypoxia sensor - HIF- $1 \alpha$ in VEGF gene activation. Implications for angiogenesis and tissue injury healing. Curr Med Chem 19(1):90-97

21. Crisostomo PR, Markel TA, Wang Y, Meldrum DR (2008) Surgically relevant aspects of stem cell paracrine effects. Surgery 143(5):577-581

22. Imtiyaz HZ, Simon MC (2010) Hypoxia-inducible factors as essential regulators of inflammation. Curr Top Microbiol Immunol 345:105-120

23. Wang M, Crisostomo PR, Herring C, Meldrum KK (2006) Human progenitor cells from bone marrow or adipose tissue produce VEGF, HGF, and IGF-I in response to TNF by a p38 MAPKdependent mechanism. Am J Physiol Regul Integr Comp Physiol 291(4):R880-R884

24. Fehrer C, Brunauer R, Laschober G, Unterluggauer H, Reitinger S, Kloss F et al (2007) Reduced oxygen tension attenuates differentiation capacity of human mesenchymal stem cells and prolongs their lifespan. Aging Cell 6(6):745-757

25. Giacoppo S, Thangavelu SR, Diomede F, Bramanti P, Conti P, Trubiani O, Mazzon E (2017) Anti-inflammatory effects of hypoxia-preconditioned human periodontal ligament cell secretome in an experimental model of multiple sclerosis: a key role of IL-37. FASEB J 31(12):5592-5608

26. Letts JA, Sazanov LA (2017) Clarifying the supercomplex: the higher order organization of the mitochondrial electron transport chain. Nat Struct Mol Biol 24(10):800-808

27. Evstafieva AG, Garaeva AA, Khutornenko AA, Klepikova AV, Logacheva MD, Penin AA et al (2014) A sustained deficiency of mitochondrial respiratory complex III induces an apoptotic cell death through the p53-mediated inhibition of pro-survival activities of the activating transcription factor 4. Cell Death Dis 5:e1511

28. Trotta AP, Gelles JD, Serasinghe MN, Loi P, Arbiser JL, Chipuk JE (2017) Disruption of mitochondrial electron transport chain function potentiates the pro-apoptotic effects of MAPK inhibition. J Biol Chem 292(28):11727-11739

29. Brown G, Borutaite V (2008) Regulation of apoptosis by redox state of cytochrome c. Biochim et Biophys Acta 1777(7-8):877-881

30. Hüttemann M, Helling S, Sanderson TH, Sinkler C, Samavati L, Mahapatra G et al (2012) Regulation of mitochondrial respiration and apoptosis through cell signaling: cytochrome $c$ oxidase and cytochrome $c$ in ischemia/reperfusion injury and inflammation. Biochim et Biophys Acta 1817(4):598-609

Publisher's Note Springer Nature remains neutral with regard to jurisdictional claims in published maps and institutional affiliations. 


\section{Affiliations}

\section{Andrey Alexandrovich Temnov ${ }^{1,2} \cdot K_{0}$ Kontantin Arkadevich Rogov ${ }^{3}$. Alla Nikolaevna Sklifas ${ }^{1}$. Elena Valerievna Klychnikova ${ }^{4}$ Markus Hartl ${ }^{5} \cdot$ Kristina Djinovic-Carugo $^{5}$ - Alexej Charnagalov ${ }^{6} \mathbb{D}$}

Andrey Alexandrovich Temnov

aa-temnov@yandex.ru

Konstantin Arkadevich Rogov

karogov@mail.ru

Alla Nikolaevna Sklifas

sklifas@mail.ru

Elena Valerievna Klychnikova

klychnikovaev@mail.ru

Markus Hartl

markus.hartl@univie.ac.at

Kristina Djinovic-Carugo

kristina.djinovic@univie.ac.at

1 Institute of Cell Biophysics RAS, Pushchino, Russia
2 Moscow Institute of Physics and Technology (State University), Dolgoprudny, Russia

3 Research Institute of Human Morphology, Moscow, Russia

4 GBUZ "Sklifosovsky Research Institute for Emergency Medicine DZ, Moscow, Russia

5 Max F.Perutz Laboratoriers, University of Vienna, Vienna, Austria

6 Institute of Science and Technology (IST Austria), Klosterneuburg, Austria 